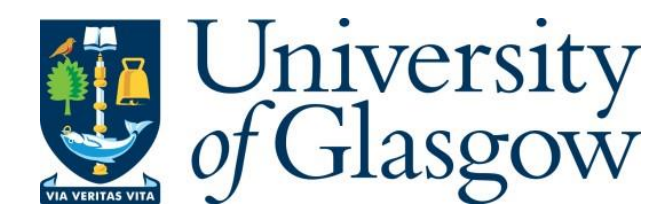

Wu, A., Liu, C., Liang, F., Zou, X., Wang, Y., Luan, P. and Li, C. (2020) Calibration on the fly - a novel two-port S-parameter measurement method for on-wafer leaky systems. IEEE Transactions on Microwave Theory and Techniques, (doi:

10.1109/TMTT.2020.2988461).

There may be differences between this version and the published version. You are advised to consult the publisher's version if you wish to cite from it.

http://eprints.gla.ac.uk/213723/

Deposited on: 15 April 2020

Enlighten - Research publications by members of the University of Glasgow http://eprints.gla.ac.uk 


\title{
Calibration on the Fly-A Novel Two-port S-Parameter Measurement Method for On-wafer Leaky Systems
}

\author{
Aihua Wu, Chen Liu, Faguo Liang, Xuefeng Zou, Yibang Wang, \\ Peng Luan, Chong Li, Senior Member, IEEE, and Nick Ridler, Fellow, IEEE
}

\begin{abstract}
In this paper, we present a two-port on-wafer scattering parameter measurement method to tackle the issue of crosstalk between probes. The proposed method treats the crosstalk separately during the system calibration and the device measurement stages, because the crosstalk during these stages is often different due to changes in the measurement conditions after the probes have been calibrated. For example, devices under test (DUTs) and calibration standards are often situated on different substrates, or, the distance between probes during calibration is different from that during DUT measurement. Based on this concept, we develop a new error model in which the crosstalk is treated as a standalone two-port error network in parallel with the two-port calibration standards or DUTs. The two-port crosstalk error generated during probing, $E_{\mathrm{CT}}$, is removed in the system calibration and corrected during the measurement of the DUT by using a dummy pair of open-circuit standards that are fabricated on the same substrate as the DUT. Since the crosstalk is corrected while measuring the DUT, rather than during system calibration, we call this method "calibration on the fly" (COF). The method is demonstrated using measurements of a 10-dB attenuator between $140 \mathrm{GHz}$ and $220 \mathrm{GHz}$.
\end{abstract}

Index Terms-Millimeter-wave measurement, on-wafer measurement, calibration, scattering parameter, error model.

\section{INTRODUCTION}

$\mathrm{O}$ VER the past 30 years, system error models for scattering parameter (S-parameter) measurement have been developed and implemented in coaxial, waveguide, and on-wafer measurement systems. The most widely used

Manuscript received xx xx, xx; revised xx xx, xx; accepted xx xx, xx. Date of publication $\mathrm{xx} \mathrm{xx}, \mathrm{xx}$; date of current version $\mathrm{xx} \mathrm{xx}, \mathrm{xx}$. This work was supported by the Research Project F2019516009 "Research on S-parameter Measurement Model and Calibration Method for $140 \mathrm{GHz} 220 \mathrm{GHz}$ " through the Natural Science Foundation of Hebei Province. (Corresponding author: Chong Li.)

A. Wu, C. Liu, F. Liang, Y. Wang and P. Luan are with the Department of Metrology and Maintenance, Hebei Semiconductor Research Institute, Shijiazhuang, 050051 China (e-mail: aihua.wu@139.com; liuchen0209@ foxmail.com; liangdao80@163.com; wangyibang_hsri@163.com; 13673114370@163.com).

$\mathrm{X}$. Zou is with the Department of Technology, Hebei Semiconductor Research Institute, Shijiazhuang, 050051 China (e-mail: zouxf999@sina.com).

C. Li is with the Division of Electronics and Nanoscale Engineering, University of Glasgow, Glasgow, G12 8LT U.K. (e-mail: chong.li@ glasgow.ac.uk).

N. Ridler is with the National Physical Laboratory, Teddington, TW11 0LW U.K. (e-mail: nick.ridler@npl.co.uk).

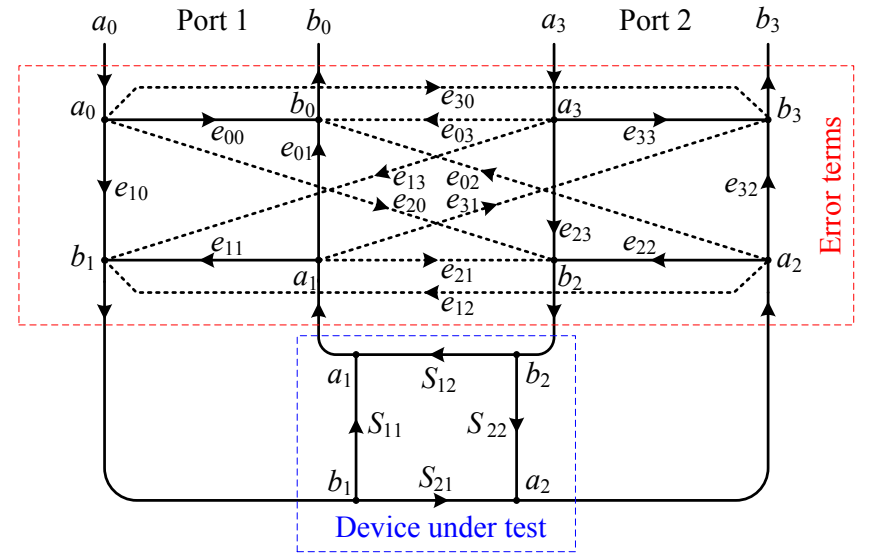

Fig. 1. The 16-term error model introduced by Speciale [4]. The solid lines represent the actual signal transmission and reflection paths; the dotted lines represent the leakages or crosstalk.

calibration methods, such as short-open-load-thru (SOLT) [1], thru-reflect-line (TRL) [2] and line-reflect-match (LRM) [3], are based on either 8-term or 12-term error models and do not contain corrections for crosstalk, because it is either non-existent or negligibly small.

For on-wafer measurements, the limitations in using the conventional error models become significant at high frequencies e.g., $100 \mathrm{GHz}$ and above. This is mainly due to the nonnegligible crosstalk or leakage generated when probes are brought closer together in order to reduce system losses at these frequencies. The fringing effect between the probes leads to a leakage path from one probe to the other when the probes are in close proximity with each other. This is the case when testing components and transistors in monolithic millimeter wave integrated circuits.

The presence of crosstalk in S-parameter measurements is a well-documented problem. To address the probe-to-probe coupling issue, Speciale [4] introduced a 16-term error model, as shown in Fig. 1. The eight conventional error terms (plotted with solid lines in Fig. 1) and eight crosstalk error terms (plotted with dotted lines) are treated as a four-port network in cascade between the vector network analyzer (VNA) and the device under test (DUT). The 16 errors can be solved by using at least five two-port standards whose S-parameters are fully known, and at least one of them is asymmetric (e.g., an 
open-load pair [5], [6]). Since then, several developments have been proposed to improve and optimize the original 16-term error model.

In 1997, Silvonen [7] developed a thru-match-reflect/ line-match-reflect (TMR/LMR) self-calibration method which reduces the number of calibration standards and therefore simplifies the calibration procedure. In 2012, a short-open-load-reciprocal (SOLR) calibration method for multi-port on-wafer measurement was introduced [8]. Subsequently, a method enabling the calibration of the full 16-term errors was developed using only four calibration standards [9]. In 2014, Dahlberg et al. [10] proposed to define the calibration standards for the line-reflect-reflect-match (LRRM) method in a reciprocal 16-term error network. More recently, Williams et al. [11] used the 16-term error model as a second-tier calibration to determine the crosstalk error terms (plotted with dotted lines in Fig. 1) provided that the other eight error terms had been solved by a multiline-TRL calibration [12], [13]. In 2018, Liu et al. [14] showed that two leakage paths i.e., $e_{21}$ and $e_{12}$, actually represent the probe-to-probe crosstalk, and the other six error terms are negligibly small and so can be ignored. In all previous cases, the crosstalk generated between probe tips i.e., $e_{21}$ and $e_{12}$, is always treated as a constant. However, this is generally not the case. In practice, the crosstalk changes as probe separation changes, especially at high frequencies.

To tackle this problem, we propose a new error model to represent the system errors in a modern two-port on-wafer $\mathrm{S}$-parameter measurement system. The varying probe-to-probe crosstalk is considered as a standalone two-port error network in parallel with any two-port standard or DUT. Since the crosstalk generated during system calibration and device measurement stages is different, it is treated separately. We first remove the crosstalk error in the system calibration and then correct the crosstalk generated during the device measurement stage. We therefore call this method "calibration on the fly" (COF).

In addition, we will limit our study to coplanar waveguide (CPW) with ground-signal-ground topology in this paper. Other topologies such as leakage in multimode waveguides for multiport or differential measurements [15], [16] are beyond the scope of this work.

\section{II.PROBE-TO-PROBE CROSSTALK}

In Speciale's 16-term error model [4], crosstalk is treated as a constant and corrected during system calibration. However, the crosstalk often changes as the measurement environment changes whether it is for off-chip calibration, where DUTs and calibration standards are on different substrates, or for on-chip calibration where DUTs and calibration standards are on the same substrate. For the former, different substrates have different dielectric properties therefore different coupling; for the latter, the distance between probes during calibration is often different from that during the measurement of the DUTs. This also applies to the off-chip calibration scenario. To demonstrate how crosstalk is affected by the conditions of the measurement, we undertook the two experiments described

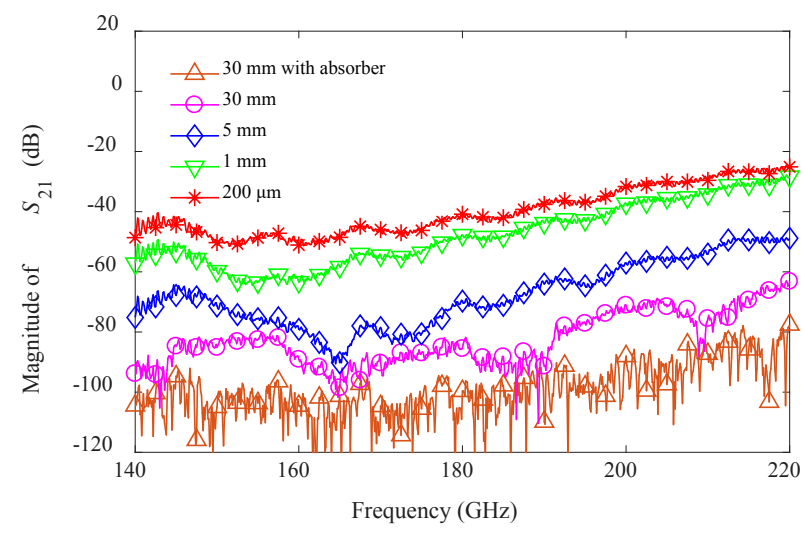

Fig. 2. Measured uncorrected raw data of $\left|S_{21}\right|$ for different separations between probes while probe tips are in the air. Probe separation has a large impact on crosstalk [14].

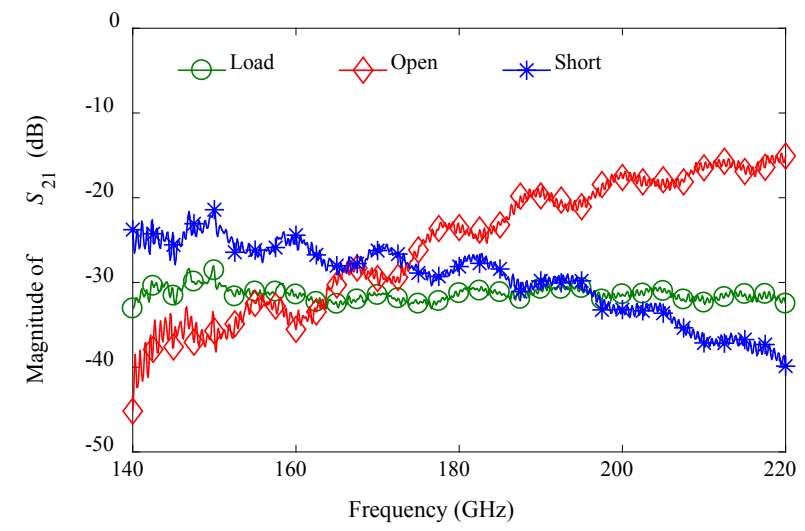

Fig. 3. Measured uncorrected raw data of $\left|S_{21}\right|$ for DUTs with the same separation and different reflection. The reflection has a large impact on crosstalk.

below.

In the first experiment, we measured raw (uncorrected) forward transmission coefficients $\left(S_{21}\right)$, which represents the crosstalk, between two G-band (140 GHz to $220 \mathrm{GHz}$ ) probes when placed in air and separated by various distances. As shown in Fig. 2, $\left|S_{21}\right|$ is close to $-20 \mathrm{~dB}$ at $220 \mathrm{GHz}$ when the probes were separated by $200 \mu \mathrm{m}$ and decreases as the distance between the probes increases. When the two probes are separated by $30 \mathrm{~mm}$, the coupling is as low as $-80 \mathrm{~dB}$, and even $-100 \mathrm{~dB}$ when a microwave absorber (Cascade PN 116-344) is inserted between the probes. From this experiment, we conclude that the crosstalk varies greatly with the distance between probes.

In another experiment we investigated how probe-to-probe crosstalk is influenced by the type of DUT being measured. To do this, we measured three pairs of standards - open-open, short-short, and load-load - on a commercial CS-15 impedance standard substrate (ISS) from GGB Industries, Inc. Each pair has the same separation distance i.e., $150 \mu \mathrm{m}$. The measured $\left|S_{21}\right|$ is plotted in Fig. 3. From these results, we can see that the crosstalk changes with both frequency and the type of DUT. The reflection coefficient of the DUTs has a significant influence on the crosstalk. Therefore, we conclude that it is 


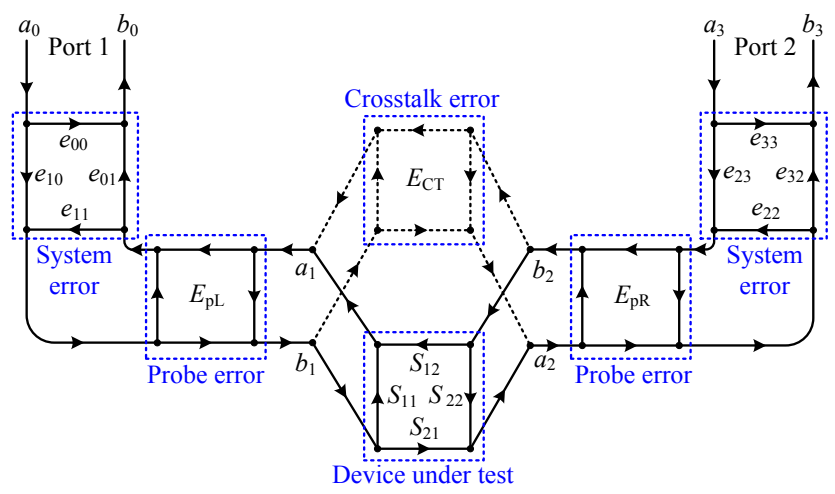

Fig. 4. The proposed new error model described in this paper. The crosstalk errors (plotted with dotted lines) are treated as a two-port network in parallel with DUT.

inappropriate to treat crosstalk for calibration and measurement as a constant.

To truly represent the crosstalk, we propose a new error model. As shown in Fig. 4, the probe-to-probe crosstalk is treated as a standalone two-port error network in parallel with the DUT during measurement. The crosstalk error, generated when probing, is removed during the system calibration and characterized using a dummy open-open pair that are fabricated on the same substrate as the DUTs. Then the crosstalk can be removed from the DUT measurements.

\section{The Proposed ERror Model}

As discussed in the previous section, crosstalk exists between probes in a two-port on-wafer S-parameter measurement system due to signals leaking from one probe tip to the other both in the substrate and in the air. The crosstalk varies depending on the loads being probed. We treat the crosstalk as a "virtual two-port network" (e.g., as an attenuator with high attenuation and high impedance) which is in parallel with a DUT during measurement, or a pair of standards during calibration. As shown in Fig. 4, all errors in the error model can be decomposed into three types: system errors, probe errors and crosstalk errors. This assumption is based on a modern VNA which has very low internal leakages [17]. The S-parameters of the "virtual network" are marked as $E_{\mathrm{CT}, i j}$, where $i, j=1$ or 2 , separately. If there is no crosstalk, the "virtual network" can be treated as an ideal pair of ideal open standards i.e., $E_{\mathrm{CT}, 11}=E_{\mathrm{CT}}$, ${ }_{22}=1$, and $E_{\mathrm{CT}, 21}=E_{\mathrm{CT}, 12}=0$. In this case, this error model becomes the conventional 8-term error model which is widely used in TRL and LRM calibration methods for coaxial and rectangular waveguides.

To apply this error model to a real on-wafer S-parameter calibration, we need to separate the crosstalk, or the "virtual network", from the DUT. Fig. 5 illustrates a possible implementation of the new technique at frequencies from 140 $\mathrm{GHz}$ to $220 \mathrm{GHz}$ (G-band). We define the reference planes at each waveguide port as Plane I and Plane II, respectively, and the reference planes at the probe tips as Plane III and Plane IV, respectively.

Like a two-tier calibration, the correction procedure requires

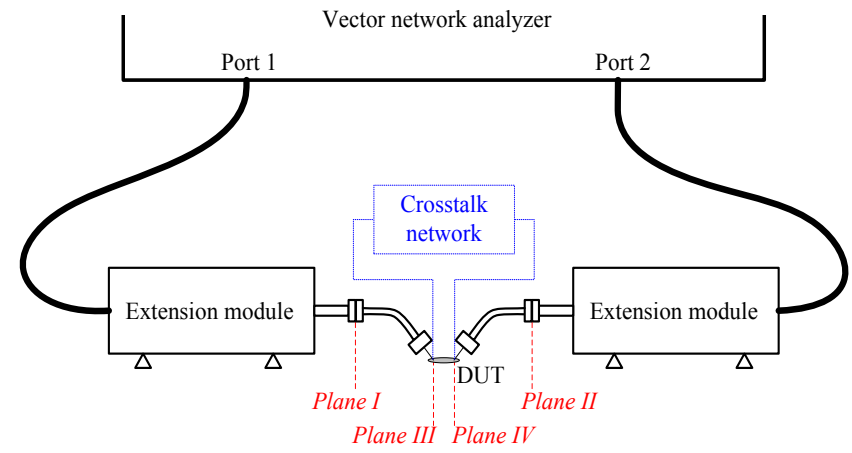

Fig. 5. Block diagram for full two-port S-parameters on-wafer measurement systems. The reference plane for each waveguide port is marked as Plane I and Plane II, respectively, and the reference plane for each probe tip is marked as Plane III and Plane IV, respectively.

two calibrations. The first calibration is a waveguide calibration, which is performed at Plane I and Plane II. Since there is no crosstalk between two waveguide ports, a standard SOLT calibration in waveguide can be implemented. The second calibration, i.e., probe calibration, is performed at Plane $I I I$ and Plane IV, which are probe tips to remove probe errors, therefore an ISS is used. To characterize the crosstalk generated when measuring DUTs, an open-open pair on the same wafer as the DUTs and having the same physical length as the DUTs is required. A detailed calibration procedure, showing how the errors in the new model are solved, is described below.

\section{A. Waveguide Calibration}

A VNA is first calibrated as its waveguide ports (i.e., Plane I and Plane II in Fig. 5) using the conventional two-port SOLT method with waveguide standards. This calibration solves the eight system error terms, i.e., $e_{00}, e_{10}, e_{01}, e_{11}, e_{33}, e_{23}, e_{32}$ and $e_{22}$, as shown in Fig. 4.

\section{B. On-wafer Calibration}

After the two-port waveguide calibration, probes are installed and one-port SOL calibration is performed at the probe tips (i.e., Plane III and Plane $I V$ in Fig. 5) individually using a commercial ISS (e.g., CS-15 from GGB Inc.) to extract the S-parameters or probe error terms of the left probe, $E_{\mathrm{pL}}$, and the right probe, $E_{\mathrm{pR}}$. This extraction can be achieved using the built-in programme "AdaptorChar Marco" in a Keysight PNA-X VNA (all of the major VNA vendors have a similar Bauer-Penfield utility [18]). We used the models provided by the vendor for the SOL standards. More accurate models e.g., based on full-wave simulation of SOL standards [19]-[20] can be used for the extraction. In addition, the SOL method can be replaced with an over-determined set of offset shorts for probe characterization [21]. Note when performing one-port SOL calibration on one probe, the other probe should be separated by at least $30 \mathrm{~mm}$ to avoid probe-to-probe crosstalk. Once the S-parameters of the two probes have been obtained, the reference planes can be moved from Planes I and II to Planes $I I I$ and $I V$ using de-embedding techniques. Details about this de-embedding process are given in the next sub-section.

In fact, Steps $A$ and $B$ can be combined using an on-wafer 
SOLR calibration method which requires an additional thru.

\section{Crosstalk Characterization}

The crosstalk errors are corrected with a dummy open-open pair with the same physical length as the DUT fabricated on the same wafer. This is because open standards, as shown in Fig. 3, have increased crosstalk as the frequency increases and are believed to be the main source of the coupling between probes. If the open-open pair standard is ideal i.e., $\left|S_{11}\right|=\left|S_{22}\right|=1$, and $\left|S_{21}\right|=\left|S_{12}\right|=0$, the measured S-parameters are the cascaded S-parameters of the left probe, crosstalk network, and the right probe. In reality, the open-open pair is nonideal and so its S-parameters $\left(S_{\text {open }}\right)$ can be defined using [22]. Based on the waveguide calibration, the measured $S$-parameters $\left(S_{\mathrm{M}}\right)$ are the cascaded S-parameters of the left probe, the crosstalk network in parallel with the open-open pair standard, and the right probe.

T-parameters are used to de-embed the left probe and the right probe from $S_{\mathrm{M}}$ [23]. The S-parameters of the open-open pair in parallel with the crosstalk network $\left(S_{\text {open } \| \mathrm{CT}}\right)$ can then be obtained. (Here the "\|l" sign means "in parallel".)

Converting S-parameters to Y-parameters, and then using (1)

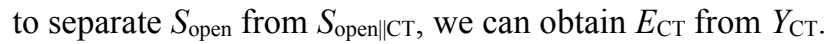

$$
Y_{\mathrm{CT}}=Y_{\text {open } \| \mathrm{CT}}-Y_{\text {open }}
$$

The relationship between S-parameters and Y-parameters is given in (2) and (3) [23], where

$$
\begin{aligned}
& \Delta_{Y} S=\left(1+S_{11}\right)\left(1+S_{22}\right)-S_{21} S_{12} \\
& \Delta Y=\left(Y_{0}+Y_{11}\right)\left(Y_{0}+Y_{22}\right)-Y_{21} Y_{12}
\end{aligned}
$$

where $Y_{0}$ is the system admittance (i.e., the inverse of the system impedance, $Z_{0}$ ).

We also investigated short-short and load-load pair standards at lower frequencies i.e., below $50 \mathrm{GHz}$, and found that the load-load pair standard has similar effect as the open-open pair standard; however, the short-short pair standard is not feasible due to a singularity generated in (1). The singularity could perhaps be mitigated using a mathematical means.

\section{DUT Test}

Also, based on the waveguide calibration, the S-parameters obtained between Plane I and Plane II for a DUT are labelled as $S_{\mathrm{m}}$. Again, using T-parameters to de-embed $E_{\mathrm{pL}}$ and $E_{\mathrm{pR}}$ from $S_{\mathrm{m}}$, the S-parameters of the DUT in parallel with the crosstalk network $\left(S_{\mathrm{DUT} \| \mathrm{CT}}\right)$ can be obtained. Then, using Y-parameters to separate the crosstalk network from $S_{\mathrm{DUT} \| \mathrm{CT}}$ in (6), the S-parameters of the DUT are obtained from $Y_{\text {DUT }}$.

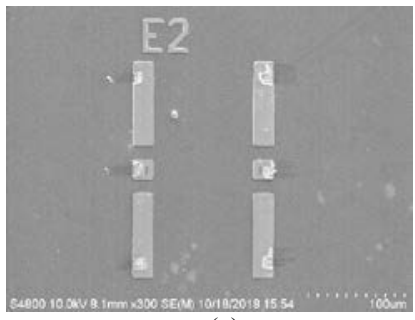

(a)

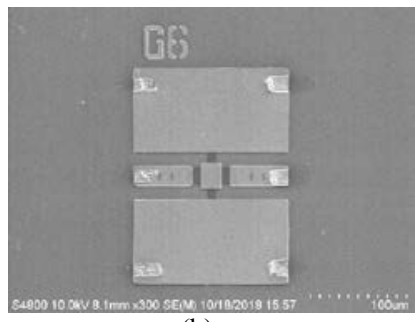

(b)
Fig. 6. SEM image of the open-open pair standard (a) and the attenuator (b). The open-open pair standard and the attenuator were fabricated on the same 4-inch semi-insulating gallium arsenide substrate using a standard photolithography method.

TABLE I

SYSTEM CONFIGURATION

\begin{tabular}{ll}
\hline \hline System Configuration & Model \& Manufacturer / Parameters \\
\hline VNA & PNA-X N5247A, Keysight \\
Frequency Extenders & WR-05, VDI \\
Probe station & Customized Cascade (manual) \\
Probes & $220-$ GSG-75-BT-M, GGB \\
No. of frequency points & 801 \\
IF bandwidth & $100 \mathrm{~Hz}$ \\
\hline \hline
\end{tabular}

$$
Y_{\mathrm{DUT}}=Y_{\mathrm{DUT} \| \mathrm{CT}}-Y_{\mathrm{CT}}
$$

When measuring other DUTs of different lengths, the crosstalk network will change and will need to be re-characterized. In this case, a corresponding open-open pair with the same length as the new DUT is required and the same crosstalk characterization procedure described in sub-section $\mathrm{C}$, above, should be implemented for the actual S-parameters of the new DUT.

According to the above method, the crosstalk error is removed while measuring the DUT rather than during the system calibration - hence, this method is called "calibration on the fly" (COF).

\section{EXPERIMENTAL RESULTS}

To evaluate the COF method, a $10-\mathrm{dB}$ attenuator was designed with the aid of commercial software (i.e., CST Microwave Studio) and fabricated on a $600-\mu \mathrm{m}$ thick semi-insulating gallium arsenide substrate using standard photolithography technology. A 400-nm layer of gold was deposited for the conductors and a thin layer of nickel-chrome alloy was used for the resistors. A two-port open-open pair was also fabricated on the same substrate. The spacing between circuits was kept to a minimum of $3 \lambda_{\mathrm{g}}$, which is greater than that suggested in [24]. The substrate was then thinned down to 100

$$
\begin{gathered}
{\left[\begin{array}{ll}
Y_{11} & Y_{12} \\
Y_{21} & Y_{22}
\end{array}\right]=\frac{Y_{0}}{\Delta_{Y} S}\left[\begin{array}{cc}
\left(1-S_{11}\right)\left(1+S_{22}\right)+S_{21} S_{12} & -2 S_{12} \\
-2 S_{21} & \left(1+S_{11}\right)\left(1-S_{22}\right)+S_{21} S_{12}
\end{array}\right]} \\
{\left[\begin{array}{ll}
S_{11} & S_{12} \\
S_{21} & S_{22}
\end{array}\right]=\frac{1}{\Delta Y}\left[\begin{array}{cc}
\left(Y_{0}-Y_{11}\right)\left(Y_{0}+Y_{22}\right)+Y_{21} Y_{12} & -2 Y_{12} Y_{0} \\
-2 Y_{21} Y_{0} & \left(Y_{0}+Y_{11}\right)\left(Y_{0}-Y_{22}\right)+Y_{21} Y_{12}
\end{array}\right]}
\end{gathered}
$$



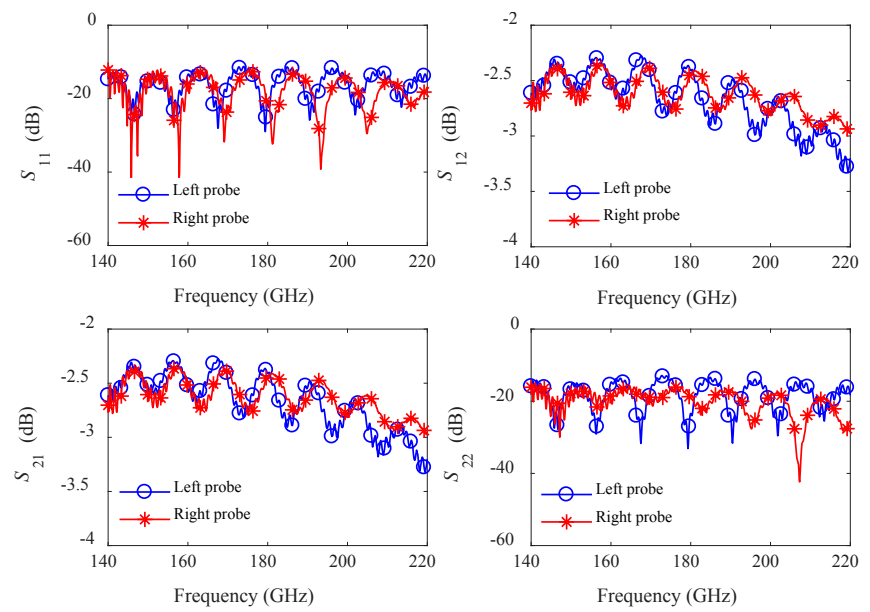

Fig. 7. S-parameters of the probes. Ports 1 and 2 are the waveguide port and probe tip, respectively.
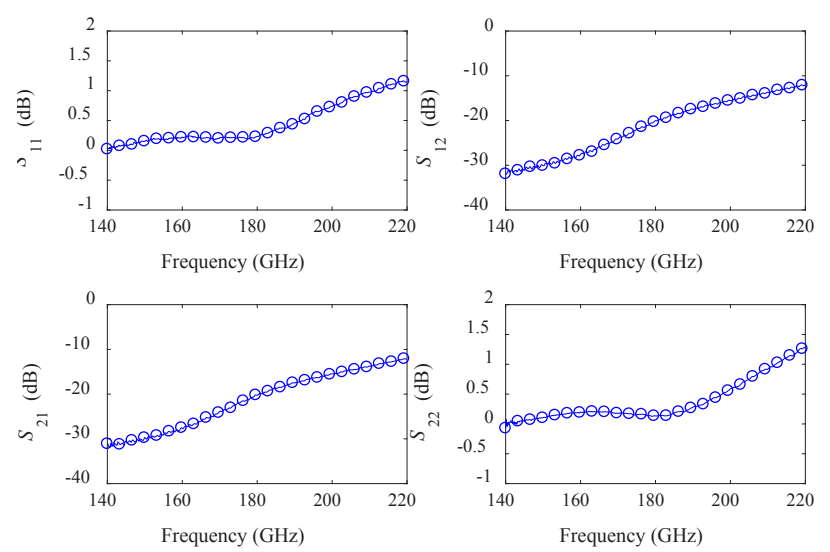

Fig. 8. S-parameters of the crosstalk network.
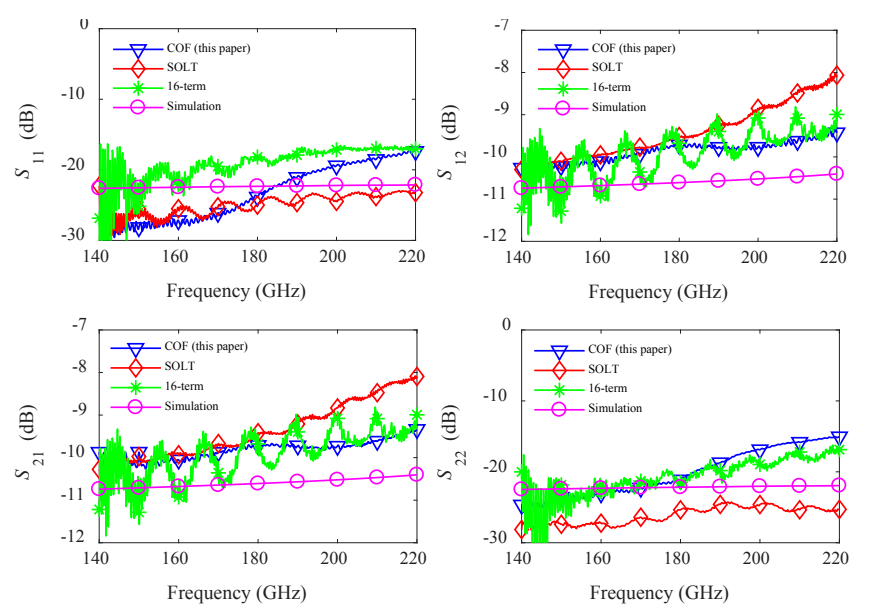

Fig. 9. Comparisons of S-parameters of a 10-dB attenuator corrected using the COF method (described in this paper), 16-term error model based on SVD method [6], SOLT (conventional 12-term error model), along with the simulated results.

$\mu \mathrm{m}$ after all circuits were made.

Fig. 6 shows a scanning electron microscope (SEM) image of the fabricated attenuator. All aforementioned components have the same edge-to-edge distance (i.e., $160 \mu \mathrm{m}$ ) in order to keep the distance constant during calibration and measurement. We defined the offset of the standards with reference to [22]. A G-band (i.e., $140 \mathrm{GHz}$ to $220 \mathrm{GHz}$ ) on-wafer S-parameter measurement setup, including a manual probe station, at the National Physical Laboratory (NPL), U.K. and two probes from GGB Industries, Inc., was used for the measurements. The system configuration is shown in Table I.

Fig. 7 shows the extracted S-parameters of the probes. Port 1 and Port 2 refer to the waveguide port and probe tip of the probes, respectively. Fig. 8 shows extracted S-parameters of the crosstalk network $\left(S_{\mathrm{CT}}\right)$ using the above-mentioned calibration procedure. As shown in this figure, the transmission coefficients (i.e., $\left|S_{21}\right|$ and $\left.\left|S_{12}\right|\right)$ are approximately $-30 \mathrm{~dB}$ at $140 \mathrm{GHz}$, increasing to close to $-10 \mathrm{~dB}$ when the frequency reaches $220 \mathrm{GHz}$. Port reflections (i.e., $\left|S_{11}\right|$ and $\left|S_{22}\right|$ ) are greater than $0 \mathrm{~dB}$. This may result from probe launch differences between calibration and measurement [25]-[27].

Fig. 9 shows S-parameters of the $10-\mathrm{dB}$ attenuator, corrected using the COF method, 16-term error model based on SVD method [6], and the standard on-wafer SOLT calibration method, along with simulated S-parameters using CST Microwave Studio. It is clear that the magnitudes of $S_{21}$ and $S_{12}$ corrected by the COF method show better agreement with the simulation results compared with the results corrected using the SOLT method, particularly at the higher frequencies in the band (i.e., above $180 \mathrm{GHz}$ ) where the presence of crosstalk is more likely to be a problem. The main reason for this is that the conventional 12-term error based SOLT calibration technique does not correct for the effect of crosstalk properly; therefore, the crosstalk contributes to the total observed transmission between the probes.

It is also observed that the S-parameters corrected using COF method is free of ripples comparing with those corrected using the 16-term error model. In the authors' opinion, the ripples shown in the 16-term error model are likely due to two reasons: one is that the 16-term error model treats the probe-to-probe crosstalk as a constant, but in fact it varies with the length and impedance of the DUT, as described in Section II; the other is that the 16-term error model requires five standards whose S-parameters are fully known, but in practice four standards along with SVD method are used which leads to approximation.

In addition, one may notice that the inconsistent of return loss shown in Fig. 9. In the authors' opinion, the difference in reflection may be caused by the inconsistent broadband matched standards between the chip and the rectangular waveguide. The reflection is heavily dependent on the load standard in SOLT calibration. However, it is difficult to manufacture broadband matched loads very accurately for rectangular waveguides or on-chip.

\section{V.CONCLUSION}

In this paper, we have presented a new error model for a two-port on-wafer measurement system. The new model truly 
reflects the variable probe-to-probe crosstalk that is subject to change during the calibration/measurement process. The new error model separates the probe errors from the system errors and treats the probe-to-probe crosstalk as an error network in parallel with the DUT. Thus, the crosstalk can be corrected while measuring the DUT. Based on the new error model, a novel COF calibration and measurement method has been presented. To implement this method, an open-open pair standard fabricated on the same substrate as the DUT was used for crosstalk correction. A load-load pair standard can also be used but not a short-short pair standard as it leads to a singularity when solving the equations. To test the method, a 10-dB attenuator was measured using a G-band on-wafer probe system. The results showed that the correction using the new error model provide improvement by almost 1-dB (i.e., 10\%) compared with the conventional SOLT method.

\section{ACKNOWLEDGMENT}

The authors would like to thank Dr. X. Shang at National Physical Laboratory, Teddington, U.K. for providing access to the wafer test equipment used in this paper.

\section{REFERENCES}

[1] J. Fitzpatrick, "Error models for systems measurement," Microw. J., vol. 21, no.5, pp. 63-66, May 1978.

[2] G. F. Engen and C. A. Hoer, "Thru-reflect-line: An improved technique for calibrating the dual six-port automatic network analyzer," IEEE Trans. Microw. Theory Techn., vol. 27, no. 12, pp. 987-993, Dec. 1979.

[3] A. Davidson, K. Jones, and E. Strid, "LRM and LRRM calibrations with automatic determination of load inductance," in Proc. 36th ARFTG Microw. Meas. Conf., Monterey, CA, USA, Nov. 1990, pp. 57-63.

[4] R. A. Speciale, "A generalization of the TSD network-analyzer calibration procedure, covering n-port scattering-parameter measurements, affected by leakage errors," IEEE Trans. Microw. Theory Techn., vol. 25, no. 12, pp. 1100-1115, Dec. 1977.

[5] K. J. Silvonen, "Calibration of 16-term error model (microwave measurement)," Electron. Lett., vol. 29, no. 17, pp. 1544-1545, Aug. 1993.

[6] K. Silvonen, "New five-standard calibration procedures for network analyzers and wafer probes," Circuit Theory Lab., Helsinki Univ. Technol., Espoo, Finland, Tech. Rep. CT-19, Mar. 1994. [Online]. Available: http://www.aplac.hut.fi

[7] K.Silvonen, "LMR 16-a self-calibration procedure for a leaky network analyzer," IEEE Trans. Microw. Theory Techn., vol. 45, no. 7, pp. 1041-1049, Jul. 1997.

[8] M. Schramm, M. Hrobak, J. Schür, and L. P. Schmidt, "A SOLR calibration procedure for the 16-term error model," in Proc. 42nd Eur. Microw. Conf., Amsterdam, The Netherlands, Oct./Nov. 2012, pp. 589-592.

[9] K. Silvonen, K. Dahlberg, and T. Kiuru, "16-term error model in reciprocal systems," IEEE Trans. Microw. Theory Techn., vol. 60, no. 11, pp. 3551-3558, Nov. 2012.

[10] K. Dahlberg and K. Silvonen, "A method to determine LRRM calibration standards in measurement configurations affected by leakage," IEEE Trans. Microw. Theory Techn., vol. 62, no. 9, pp. 2132-2139, Sep. 2014.

[11] D. F. Williams, F. Schmuckle, R. Doerner, G. N. Phung, U. Arz, and W. Heinrich, "Crosstalk corrections for coplanar-waveguide scattering parameter calibrations," IEEE Trans. Microw. Theory Techn., vol. 62, no. 8, pp. 1748-1761, Aug. 2014.

[12] D. C. Degroot, J. A. Jargon, and R. B. Marks, "Multiline TRL revealed," in Proc. 60th ARFTG Microw. Meas. Conf., Washington, DC, USA, Dec. 2002, pp. 131-155.

[13] D. F. Williams, C. M. Wang, and U. Arz, "An optimal multiline TRL calibration algorithm," in IEEE MTT-S Int. Microw. Symp. Dig., Philadelphia, PA, USA, Jun. 2003, pp. 1819-1822.

[14] C. Liu, A. Wu, C. Li, and N. Ridler, "A new SOLT calibration method for leaky on-wafer measurements using a 10-term error model," IEEE Trans. Microw. Theory Techn., vol. 66, no. 8, pp. 3894-3900, Aug. 2018.
[15] V. Teppati and A. Ferrero, "On-wafer calibration algorithm for partially leaky multiport vector network analyzers" IEEE Trans. Microw. Theory Techn., vol. 53, no. 11, pp. 3665-3671, Nov. 2005.

[16] M. Wojnowski, V. Issakov, G. Sommer, and R. Weigel, "Multimode TRL calibration technique for characterization of differential devices" IEEE Trans. Microw. Theory Techn., vol. 60, no. 7, pp. 2220-2247, Jul. 2012.

[17] N. M. Ridler and R. G. Clarke, "Establishing traceability to the international system of units for scattering parameter measurements from $750 \mathrm{GHz}$ to $1.1 \mathrm{THz}$," IEEE Trans. THz Sci. Technol., vol. 6, no. 1, pp. 2-11, Jan. 2016.

[18] R. F. Bauer, and P. Penfield, "De-Embedding and unterminating" IEEE Trans. Microw. Theory Techn., vol. 22, no. 3, pp. 282-288, Mar. 1974.

[19] M. Spirito, L. Galatro, G. Lorito, T. Zoumpoulidis and F. Mubarak, "Improved RSOL planar calibration via EM modelling and reduced spread resistive layers," in Proc. 86th ARFTG Microw. Meas. Conf., Atlanta, GA, USA, Dec. 2015, pp. 1-5.

[20] F. Mubarak, V. Mascolo, G. Rietveld, M. Spirito, K. Daffe and K. Haddadi, "Parameterization models for traceable characterization of planar CPW SOL calibration standards," in 2018 Conf. on Precision Electromagnetic Meas. (CPEM 2018), Paris, France, Jul. 2018, pp. 1-2.

[21] T. Reck, L. Chen, C. Zhang, A. Arsenovic, C. Groppi, A. Lichtenberger, R. Weikle, and N. S. Barker, "Micromachined probes for submillimeterWave on-wafer measurements-Part II: RF design and characterization," IEEE Trans. THz Sci. Technol., vol. 1, no. 2, pp. 357-363, Nov. 2011.

[22] K. Jones and E. Strid, "Where are my on-wafer reference planes?" in Proc. 30th ARFTG Microw. Meas. Conf., Dallas, TX, USA, Dec. 1987, pp. $27-40$.

[23] D. A. Frickey, "Conversions between S, Z, Y, H, ABCD, and T parameters which are valid for complex source and load impedances," IEEE Trans. Microw. Theory Techn., vol. 42, no. 2, pp. 205-211, Feb. 1994.

[24] Franz Josef Schmückle, Thorsten Probst, Uwe Arz, Gia Ngoc Phung, Ralf Doerner and Wolfgang Heinrich, "Mutual Interference in Calibration Line Configurations," in Proc. 89th ARFTG Microw. Meas. Conf., Honololu, HI, USA, Jun. 2017, pp. 1-4.

[25] D. F. Williams and R. B. Marks, "Calibrating on-wafer probes to the probe tips" in Proc. 40th ARFTG Microw. Meas. Conf., Orlando, FL, USA, Dec. 1992, pp. 136-143.

[26] D. F. Williams and R. B. Marks, "Compensation for substrate permittivity in probe-tip calibration" in Proc. 44th ARFTG Microw. Meas. Conf., Boulder, CO, USA, Dec. 1994, pp. 20-30.

[27] D. F. Williams and D. K. Walker, "Compensation for geometrical variation in coplanar waveguide probe-tip calibration" IEEE Microw. Wireless Compon. Lett., vol. 7, no. 4, pp. 97-99, Apr. 1997. 


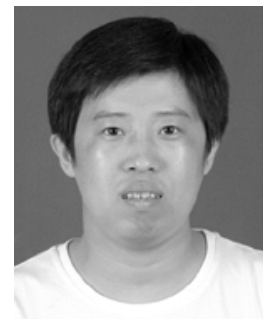

Aihua Wu was born in Zhangjiakou, China, in 1980. He received the BSc and the MEng degrees in Microelectronics from Jilin University, Changchun, China, in 2004 and 2007, respectively.

$\mathrm{He}$ joined the Hebei Semiconductor Research Institute, Shijiazhuang, China, in 2007, where he is currently a Senior Research Engineer with the Department of Metrology and Maintenance. From April to October 2017, he joined the National Physical Laboratory, Teddington, U.K., as a Visiting Researcher. His current research interests include microwave metrology, particularly on-wafer noise measurements and instrumentation, and the fabrication of on-wafer devices for ultra-wideband S-parameters and noise parameter calibration and verification.

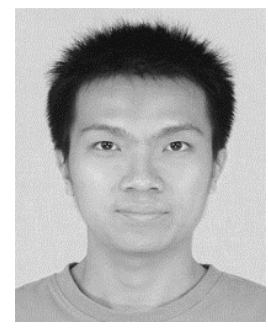

Chen Liu was born in Hengshui, China, in 1986. He received the BEng and the MEng degrees in Measuring and Testing Technologies and Instruments from Xidian University, Xi'an, China, in 2009 and 2012, respectively.

He joined the Department of Metrology and Maintenance, Hebei Semiconductor Research Institute, Shijiazhuang, China, as a Research Engineer in 2012. From April to October 2017, he joined the National Physical Laboratory, Teddington, U.K., as a Visiting Researcher. His current research interests include the design and characterizing of on-wafer SOLT and TRL S-parameters calibration kits, the development of accurate on-wafer S-parameters and noise parameter measurement techniques, and the verification of on-wafer S-parameters and noise parameter using active and passive devices.

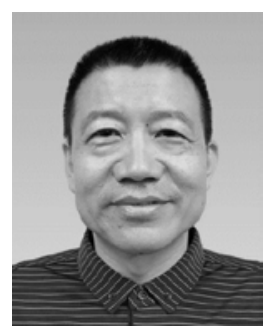

Faguo Liang was born in Liaocheng, Shangdong, China, in 1965. He received the BEng degree in microelectronics from Shangdong University, Jinan, China, in 1984, and the MEng degree in Microelectronics from Hebei Semiconductor Research Institute, Shijiazhuang, China, in 1989.

From 1984 to 1986, he was a research assistant with the Jinan Semiconductor Research Institute, Shijiazhuang, China. Since 1989, he was an Engineer with the Hebei Semiconductor Research Institute. Now, he is a professor and his research interest include microwave instrumentation metrology, on-wafer microwave parameter measurements.

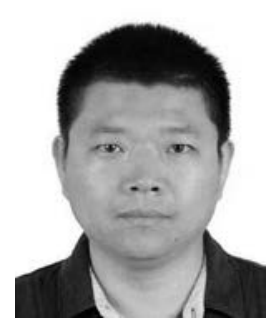

Xuefeng Zou was born in Shuangyashan, China, in 1977. He received the BEng degree in Automation from China University of Mining and Technology, Xuzhou, China, in 2000.

$\mathrm{He}$ joined Hebei Semiconductor Research Institute, Shijiazhuang, China, as a Research Engineer in 2000, where he is currently a Senior Research Engineer with the Department of Technology. His current research interests include FET devices and circuits, GaAs MMIC, GaN-based FETs, and measurement techniques.

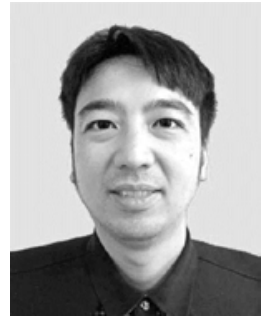

Yibang Wang was born in Jining, China, in 1987. He received the BSc degree in Communication Engineering from Nanjing University of Aeronautics, Nanjing, China, in 2009, and the MSc degree in Instrument Design from Handan Purified Equipment Research Institute, Handan, China, in 2012.

He joined the Department of Metrology and Maintenance, Hebei Semiconductor Research Institute in Shijiazhuang, China. His current research interests include microwave metrology, particularly on-wafer terahertz S-parameters measurements, and the fabrication of on-wafer calibration kits and research of calibration algorithm.

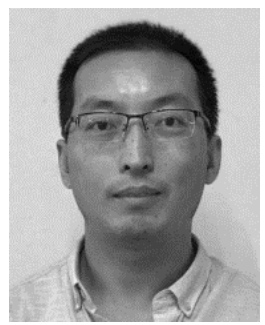

Peng Luan was born in Liaoning, China, in 1978. He received the BEng degree from North Eastern University, Shenyang, China, in 2002, and the MSc degree from University of Electronic Science and Technology of China, Chengdu, China, in 2011.

He joined the Department of Metrology and Maintenance, Hebei Semiconductor Research Institute, Shijiazhuang, China, as a Research Engineer in 2002. His current research interests include the design and characterizing of on-wafer TRL S-parameters calibration kits, the development of accurate on-wafer S-parameters and load-pull system measurement techniques, and the verification of on-wafer S-parameters and load-pull system using active and passive devices. 


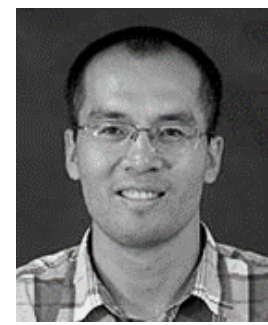

Chong Li (M'12-SM'17) was born in Liaoning, China in 1979. He received the BEng degree from Donghua University, China, in 2002, the MSc degree (Distinction) from the University of Manchester, U.K., in 2007, and the PhD degree in Electronics and Electrical Engineering from the University of Glasgow, U.K., in 2011.

He became a Postdoctoral Research Assistant in 2011 and later a Postdoctoral Research Associate at the University of Glasgow, working on development of millimetre-wave signal sources and terahertz imaging systems. He joined the National Physical Laboratory (NPL), U.K., in January 2014 as a Higher Research Scientist where he contributed to and led several commercial projects and U.K. national and European research projects. He was the measurement service provider (MSP) of the ultrafast waveform metrology service at NPL. He also led work on microwave and millimetre-wave on-wafer measurements. He became a lecturer at the University of Glasgow in August 2017 and is currently leading the Microwave and Terahertz Electronics group. His current research interests include microwave and terahertz components, systems and metrology and next generation wireless communications.

Dr. Li is an Associate Editor of Royal Society Open Science and has published more than 60 journal and conference papers. Dr. Li held a visiting position at the Advanced Technology Institute (ATI), University of Surrey in 2017 and won the Best Non-student Paper Prize at Loughborough Antennas and Propagation Conference (LAPC) in 2015.

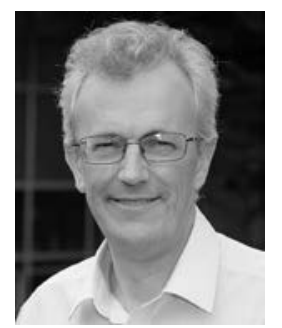

Nick Ridler (M'03-SM'06-F'14) received the BSc degree from King's College, University of London, U.K., in 1981. He has more than 35 years' experience working in industrial, government and academic research establishments. He is currently Head of Science for the Department of Electromagnetic and Electrochemical Technologies at the UK's National Physical Laboratory. $\mathrm{He}$ is also Non-Executive Director of LA Techniques Ltd, and a Visiting Professor at the University of Leeds (Pollard Institute), the University of Liverpool (Department of Electrical Engineering and Electronics) and the University of Surrey (Faculty of Engineering and Physical Sciences). He is a Fellow of the Institution of Engineering and Technology (IET) and a Fellow of the Institute of Physics (IOP). He is a past Chair of the IEEE MTT Society's "Microwave Measurements" Technical Committee, and, Past President of ARFTG (the Automatic RF Techniques Group). His main area of interest is precision high-frequency electromagnetic measurement $(1 \mathrm{kHz}$ to $1 \mathrm{THz})$. 